\title{
Cortázar y Poe, las lecturas de los maestros
}

\author{
Cortázar e Poe, as leituras dos mestres
}

\author{
LEONARDO GIL GOMEZ* \\ Northwestern University, Evanston, Illinois, Estados Unidos
}

\begin{abstract}
Resumo: O seguinte artigo propõe uma relação entre a crítica literária e a escrita criativa. Parte da ideia de que o rigor do critico deveria ser o primeiro passo que o artista deve dar para desenvolver as habilidades necessárias no processo de escritura. Assim, as leituras do escritor devem buscar na tradição literária os elementos que dão forma a seu próprio trabalho literário. Este ensaio segue a tradução e a análise que Julio Cortázar fez da obra do E. A. Poe. Através delas, o argentino pôde identificar as principais ideias da contística de Poe (brevidade, economia de recursos, unidade de impressão, intensidade e tensão). Anos depois, essas categorias foram utilizadas pelo próprio Cortázar em suas obras. Como um passo além da noção da literatura de Poe, Cortázar utilizou essas ideias para expressar suas preocupações sobre a escritura da obra literária. O caminho crítico e literário que este texto sugere segue uma tradição que pode ser seguida até N. Hawthorne e além da influência de Poe e Cortázar.
\end{abstract}

Palabras-chave: intensidade; tensão; estrutura narrativa; composição narrativa; Poe; Cortázar; conto; leitura; escritura.

\begin{abstract}
The following text proposes a relationship between literary criticism and creative writing. Starts from the idea that the rigor of criticism should be the first step an artist should take toward the development of the abilities needed in the creative writing process. Thus, the writer's readings should look after the elements that shape his or her own work within the literary tradition. This essay follows Julio Cortázar's translation and the analysis of E. A. Poe's work. Through them, the Argentinian could identify the main elements that shape Poe's storytelling (brevity, economy of resources, unity of effect, intensity and tension). Years later, such categories were displayed by Cortázar himself for expressing his own concerns regarding the creative process. The critic abd literary path sugested by this text, aims to propose a tradition that may be traced to N. Hawthorne and goes beyond Cortázar and Poe's influence.

Keywords: intensity; tension; narrative structure; narrative composition; Poe; Cortázar; short story; reading; writing.
\end{abstract}

* Doutorando em Espanhol e Português (Northwestern University, Estados Unidos). <leogilg@gmail.com>. 
De muchas maneras, la labor del escritor es cercana a la del crítico literario. En la medida en que necesita descifrar los mecanismos de la obra literaria para encontrar modelos creativos y formas de transmitir una forma particular de ver el mundo. En el proceso de escritura es de vital importancia entender que todo lo que creemos auténtico en nuestra experiencia probablemente ya ha sido expresado en diversas manifestaciones artísticas y que quien se proponga alcanzar una voz propia debe conocer la tradición, reconocerse en ella y, luego, tomar distancia en la búsqueda de su estilo. Por otro lado, la labor del crítico se acerca a la del artista en la medida en que es también una escritura

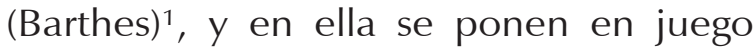
los mismos procedimientos, las mismas preguntas con relación a la tradición, el mundo y el texto, que el escritor requiere para producir su obra literaria. Es necesario aclarar aquí que entiendo la crítica no como el lugar desde el cual se legitiman (o no) las obras literarias, sino el espacio en que un lector socializa su interpretación del texto literario. Nombres que ejemplifican este punto, de un lado y del otro, tenemos de sobra: Charles Baudelaire, W. H. Auden, Kandinsky, Borges, Vargas Llosa, Octavio Paz, Alejo Carpentier, Piglia, Barthes, y un largo etcétera que incluye a quienes abordaré en este texto: Julio Cortázar y E. A. Poe.

En 1953, Julio Cortázar recibe el encargo de traducir la obra completa de Edgar Allan

\footnotetext{
1 Aunque no es el lugar para extendernos en esta discusión, se puede observar un hito del debate sobre la condición de la crítica como escritura y su relación con el arte: Roland Barthes, de quien tomo esta mirada sobre la crítica nos ofrece una muestra en uno de sus ejercicios críticos más radicales, en los que aquello que en el artista se asociaría con la inventiva o cierta capacidad creativa, ocurre con su análisis de Sarrasine, de Balzac, publicado bajo el título $S / Z$
}

Poe para la Universidad de Puerto Rico. Eran los años de París, Cortázar tenía 39 años, dos libros de cuentos publicados ( $L a$ otra orilla, 1946, con el cual no se sentía cómodo y Bestiario, 1951), acababa de contraer matrimonio con Aurora Bernárdez y, aunque había emigrado de Argentina con una beca de estudios, llevaba una vida austera en la capital francesa. Cuenta en cartas a Fredi Guntham, del 12 de mayo y el 25 de agosto del '53:

A todo esto, conseguimos dos piezas y una cocinita en una meublé de la rué de Gentilly, cerca de la Place d'Italie. Pas marrant com me quartier, mais on a les deux chambres, pleines de soleil et assez grandes, et la cuisine, pour 12.000 bailes. No es caro, si piensas que por piezas separadas pagábamos 7.000 cada uno. (...) Como creo haberte dicho, me han confiado la traducción de todas las obras en prosa de Edgar Poe, trabajo para seis meses por lo menos, ya que además hay que escribir un estudio crítico-bibliográfico. En vista de eso, largué mi empleo matinal, y me voy a Roma a trabajar allá. Parece que en Roma se pueden conseguir pequeños "studios" o departamentos por unas 20.000 liras mensuales. (...) París, a pesar de la huelga, está hermoso como siempre. (...) La huelga nos ha tenido encerrados casi veinte días, pues no había ni metro ni autobuses. Los aprovechamos para traducir, leer y caminar por el barrio. Tenemos una ducha municipal a ocho cuadras, donde te bañas espléndidamente por veinticinco francos (CORTÁZAR, 2000, p. 511).

La nueva traducción de Poe, que fue publicada en 1956, le permitió a Cortázar estudiar al cuentista norteamericano y no solo entregar una de las mejores versiones de su obra al español, sino además desentrañar los mecanismos internos de su cuentística, lo cual se verá reflejado tanto 
en la obra del propio Cortázar, como en sus reflexiones sobre el oficio.

La traducción, como sabemos es un asunto que trasciende la simple homologación semántica entre dos lenguas, y que para el caso de la literatura implica considerar variaciones de estilo, términos intraducibles, localismos, entre otros problemas. Esta característica exigió de Cortázar el rigor del crítico. No es que Cortázar fuera un novato en aquellas tareas; sus inquietudes literarias juveniles le inclinaron a la poesía y ya durante su formación como poeta había demostrado la misma solidez que su posterior y más conocido trabajo de traductor demuestra. Fruto de este periodo es el conjunto de ensayos recopilado bajo el título Imagen de John Keats.

Así, pues, se encuentra en la figura del argentino el talante necesario para emprender la tarea, no solo de traducir la obra de Poe, sino de hurgar en ella con la curiosidad del artista y luego comentarla con la autoridad crítica suficiente para señalar la precariedad del trabajo existente sobre la obra de Poe hasta el momento en que él se ocupa de su estudio (1956, p. LV-LVI). Cortázar comenta que la crítica trata, bien de interpretar la obra de Poe a la luz de circunstancias personales y psicológicas que hubieran podido condicionarla, hasta el punto de formular estudios clínicos del "caso Poe"; o bien de subestimar la obra de Poe según el gusto de la época. En ambos casos, sobrevaloración de su biografía o subestimación de su obra, señala el argentino se llega al mismo problema: "un balance de la obra de Poe y sus consecuencias, de lo absoluto y lo relativo de ella, no puede lograrse si se la reduce a un caso clínico, o a una serie de textos literarios. Hay más, hay siempre más" (ibid.).
Lo anterior atiende tanto la disciplina de Cortázar, como su capacidad para tomar distancia de las interpretaciones ofrecidas por la crítica del momento, en aras de ofrecer una perspectiva propia. Pero no solo se da cuenta de la pobre recepción de la obra de Poe y el incipiente contexto literario en el que surge su obra (por lo cual no deja de ser sorprendente el lugar que ocupa el norteamericano en la literatura universal y su influencia sobre el simbolismo, tras la versión al francés que hiciera Baudelaire); el argentino observa también, en la búsqueda de las coordenadas de la obra de Poe, la conciencia de éste respecto a su medio y la influencia que sobre él tiene la obra de Hawthorne, otro autor subestimado en su momento y cuya magnitud se refleja en la fuerte influencia sobre las letras posteriores.

Vale resaltar, pues, una línea de trabajo que va desde Hawthorne hasta Cortázar, una pequeña tradición crítica, cuyos resultados apuntan a la creación. ${ }^{2}$ Así como Poe estudió a Hawthorne para desarrollar su estética literaria, Cortázar hizo lo propio con Poe. Con la traducción y el estudio crítico de Poe, Cortázar buscó establecer los fundamentos de una cuentística, de su cuentística.

Esta influencia evoluciona hasta que el propio Cortázar se distancia del norteamericano, en un flujo que da cuenta de su crecimiento como autor. Como he dicho, tal proceso consiste en conocer los elementos de la tradición, reconocerse en ellos (es decir, reconocer su influencia) y luego desafiarlos para crear una voz propia. Reconocer al padre exige su muerte $y$ cada uno de estos autores ha aceptado y

\footnotetext{
2 O al revés, si algún lector-creador siente invertida su escala de valores: una tradición creativa que pasa por la reflexión crítica.
} 
rechazado elementos de su predecesor para poder establecer los horizontes de su obra literaria. En Cortázar, la evolución mencionada parece alcanzar su expresión más auténtica y radical en cuentos como "El perseguidor".

Este proceso inicia con la lectura de la obra de Poe y el contraste de las interpretaciones de Cortázar con los propios textos críticos del norteamericano. En su búsqueda, el argentino encuentra que buena parte de la reflexión de Poe sobre su cuentística se encuentra en la reseña que hace sobre Hawthorne. Este texto, junto con la "Filosofía de la composición", se encuentra entre los más estudiados en los talleres literarios contemporáneos que se ocupan de cuento y/o poesía.

En "Hawthorne", Poe propone una fuerte distinción entre las intenciones del cuento y las de la poesía. Sabemos por la envergadura de su obra que fue un maestro de ambos géneros y por eso sus reflexiones, aunque tiendan a disonar con lo que sobre esta distinción se dice en nuestros tiempos, conservan aspectos de gran importancia. Allí expone que el género que mejor se presta para la expresión literaria es la poesía: "un poema rimado cuya duración no exceda de una hora de lectura. Solo dentro de este límite puede alcanzarse la más alta poesía" (POE, 1973, p.134). Luego de definir la brevedad como elemento fundamental para alcanzar el efecto deseado (en el cuento, según Poe, consiste en que la duración de su lectura esté entre media hora y dos horas), hace énfasis en la unidad de efecto o impresión. Esta categoría es muy importante, tanto para la poesía como para el cuento: "Esta unidad no puede preservarse adecuadamente en producciones cuya lectura no alcanza a hacerse en una sola vez. (...) Sin unidad de impresión no se pueden lograr los efectos más profundos" (id., p.135). Este principio supone, a la vez, economía de recursos y coherencia de los elementos propuestos para la narración. Se trata de la subordinación de todos los elementos del relato a la búsqueda del efecto deseado por el autor: "Si su primera frase no tiende ya a la producción de dicho efecto, quiere decir que ha fracasado en el primer paso. No debe haber una sola palabra en toda la composición cuya tendencia, directa o indirecta, no se aplicara al designio preestablecido" (ibid., 136).

Un efecto preconcebido, brevedad y unidad de impresión; el encuentro entre estas ideas y su traductor al español da sus frutos. Lorna Valerie Williams señala que la composición del prólogo a la edición de la obra de $\mathrm{Poe}^{3}$, le permitió a Cortázar formular aspectos de su propia teoría del cuento a partir de los postulados del autor norteamericano. Algo que tiene sentido, dada su influencia en la propia cuentística de Cortázar, y el hecho de que aquel hubiera hecho lo propio con su antecesor, N. Hawthorne. Como punto de partida de la teoría del cuento que luego desarrollará Cortázar, tenemos el siguiente fragmento, que coincide casi punto por punto con los apuntes de Poe sobe Hawthorne:

Poe descubrió inmediatamente la manera de construir un cuento, de diferenciarlo de un capítulo de novela, de los relatos autobiográficos, de las crónicas romanceadas de su tiempo. Comprendió que la eficacia de un cuento depende de su intensidad como acaecimiento puro, es decir, que todo

Titulado "El poeta, el narrador y el crítico" y publicado junto a una exhaustiva biografía en el volumen de la prosa completa de Poe de la Universidad de Puerto Rico, en 1956. 
comentario al acaecimiento en sí (y que en forma de descripciones preparatorias, diálogos marginales, consideraciones a posteriori alimentan el cuerpo de la novela y de un mal cuento) debe ser radicalmente suprimido. Cada palabra debe confluir, concurrir al acaecimiento, a la cosa que ocurre, y esta cosa que ocurre debe ser solo acaecimiento y no alegoría o pretexto para generalizaciones psicológicas, éticas o didácticas. Un cuento es una máquina literaria de crear interés (CORTÁZAR, 1956, p. LXXIV).

Entre otros elementos, Cortázar se concentra en el efecto que debe tener un cuento (razón de ser del mismo, según Poe); la brevedad y la economía de recursos que ya he mencionado; y la orientación a la verdad, en oposición a la orientación a la belleza, propia del poema según Poe. Esta última no se encuentra en el fragmento citado, pero Cortázar lo trata en su prólogo, siguiendo la diferencia que ofrece Poe en "Hawthorne": "Mientras el ritmo de este último [el poema] constituye ayuda esencial para el desarrollo de la más alta idea del poema -la idea de lo Bello-, (...) Pero con frecuencia y en alto grado el objetivo del cuento es la verdad" (POE, 1976, p. 136).

Llaman la atención varios elementos de este análisis: en primer lugar, aquello que podemos identificar como economía, que en Poe tiene que ver con la unidad de impresión y que Cortázar asocia a la intensidad ${ }^{4}$ del cuento; en segundo lugar, la noción de lo narrado como acaecimiento puro; y finalmente, la relación con la verdad, término que frecuentemente resulta problemático.

4 Para Cortázar la intensidad va a adquirir un papel primordial a la hora de definir su propia poética. Se desarrollará después en "Del cuento breve y sus alrededores"
Los primeros dos elementos están asociados, según la lectura que Cortázar hace de Poe. Para Cortázar la cosa que ocurre en el cuento debe ser intensa en sí misma, y eso se relaciona tanto con el efecto que busca el cuentista, como con la forma de expresarlo. Por un lado, coincide con Poe en que nada en el cuento debe estar para adornar o embellecer el acontecimiento; y por otro, ese acontecimiento debe ser presentado como acaecimiento puro. La expresión que utiliza Cortázar es difícil de rastrear, pues no es un término de Poe y la explicación que ofrece Cortázar, si bien es suficiente para ubicarlo en la cuentística de Poe, se diluye cuando se trata de asociar a otros problemas. Williams (1974) señala que, para el argentino, se trata de plasmar la situación narrativa según la estructura más profunda de los personajes que se mueven dentro de ella. Cortázar hace énfasis en que el problema no es solo temático; cuando habla de la estructura más profunda, se refiere a que es profunda en el hombre, no a la estructura narrativa. En estos términos, el propio Cortázar hace una lista de los elementos que la componen, al menos en la obra de Poe: "Ciertos cuentos serán intensos porque enfrentan al hombre con su circunstancia en conflictos trágicos, de máxima tensión; o porque ponen en escena seres donde se concentran ciertas facultades en su punto más alto, ciertas fatalidades misteriosas, ciertas conjeturas sobrenaturales, cierto heroísmo en la procura de un fin". (CORTAZAR, 1956, p.LXXV). Pese a esta sistematización que ofrece el argentino, siguen sobre la mesa dos cartas cerradas: la verdad y otra, que suma el propio Cortázar: la circunstancia del hombre.

Aquí hay que señalar otro de los puntos de partida de la cuentística de Cortázar y su 
relación con Poe. El argentino encuentra, y lo señala en su prólogo, que el cuentista norteamericano, por diversas razones es incapaz de plantearse la pregunta por la dimensión interior del otro. Característica que se puede observar en sus relaciones interpersonales y que tendrá especial repercusión en su obra. Este rasgo particular de Poe se debe, según Cortázar, en buena parte a una constante debilidad física en contraste con una colosal fortaleza intelectual; de una parte, estaba en desventaja para desempeñar roles sociales que involucraran fuerza física y desenvoltura social (desde hacerse valer en la escuela, hasta su relación con las mujeres); de otra, Poe compensaba esta limitación con una estimación de sí mismo que lo ponía por encima de sus semejantes en virtud de sus habilidades intelectuales y su racionalismo audaz. En consecuencia, los personajes de la obra de Poe carecen de un mundo interior complejo y responden con mayor frecuencia a figuras arquetípicas (recurso, por cierto, propio de las historias de terror y de aquellas que apelan a manifestaciones de lo onírico). Así, pues, Cortázar observa que cuando se encuentra en la obra de Poe un personaje con dimensión interior compleja, con frecuencia éste tiene fuertes paralelismos con el autor, lo que le lleva a concluir que aquellos personajes son ficcionalizaciones, alter-egos, del propio Poe. Auguste C. Dupin, el detective de Poe, que se erige como paradigma de los relatos policiales, es un buen ejemplo de ello. Cortázar resalta, además, que el hecho de que en las relaciones entre los héroes de Poe y los demás personajes, el otro fuera frecuentemente descrito como alguien inferior y despreciable. Es en el otro, en quien Poe se permite anclar lo grotesco, lo horroroso y lo macabro de sus ficciones, mientras la agudeza racional caracterizaba a los personajes más cercanos a él.

En Cortázar, la pregunta por el otro se torna más compleja y está asociada al problema ya mencionado: el hombre y su circunstancia. En sus inicios, la inclinación por lo fantástico le llevó a explorar la literatura por vías similares. Bestiario se publicó en 1951, y los temas de Cortázar entonces funcionaban siguiendo modelos cercanos a Poe: intensidad, brevedad, exploración del misterio, personajes arquetípicos y, si bien en Cortázar lo perverso no tiene la fuerza de Poe, sí nos encontramos de frente con la idea de lo monstruoso. De hecho, Néstor García Canclini propone en su libro Cortázar, una antropología poética, un recorrido que parte de la noción de monstruosidad en las primeras obras de Cortázar: el minotauro y el laberinto en Los reyes, por ejemplo, o el desarrollo de lo monstruoso en todo Bestiario, hasta la búsqueda de autenticidad por parte de los personajes en Los premios, Rayuela y El perseguidor. Con García Canclini encontramos que detrás de esta exploración está la pregunta por lo humano, que Cortázar nunca disocia del cuestionamiento de la realidad. Para él toda literatura es fantástica; una distinción cartesiana entre lo real y lo fantástico no es posible en ella en virtud del pacto que nos propone. Tenemos aquí un elemento muy cercano a Poe, quien comprendía que de todos los efectos posibles de los cuentos, aquellos cercanos al horror y que comúnmente estaban asociados a lo desconocido, lo sobrenatural y lo extraordinario, eran los de mayor potencial. En estos términos, Cortázar anuncia desde sus cuentos la fragilidad del límite entre aquello que aceptaríamos como real y el mundo de lo fantástico; un límite que en 
ocasiones es asombroso, pero también oscuro y proclive al horror: "Las referencias a lo cotidiano hacen creer que estamos tranquilamente en lo inmediato, en tal calle de Buenos Aires o ante tal costumbre familiar. Pero en cuanto nos sentimos seguros, lo fantástico se filtra y hace danzar sus monstruos" (GARCÍA C. 30-31).

Su siguiente libro de cuentos, Final de juego (1956), seguirá la misma línea, no porque en ellos se repitan temas o formas, sino porque las inquietudes básicas de Cortázar encuentran suficientes variaciones para expresarse sin que por ello se vean modificados los principios básicos: el misterio, un personaje más o menos arquetípico, y una aproximación a lo fantástico que radica más en el cuestionamiento de lo que entendemos por realidad que en la configuración de un universo distinto al cotidiano. Así, pues, la pregunta por aquello que interpreta Cortázar cuando habla de "enfrentar al hombre con su circunstancia" como una de las intenciones de la literatura, se puede resolver en primera instancia mediante la puesta en escena de personajes arquetípicos (por parte de Poe) y el paso que de este punto da Cortázar hacia la pregunta por la autenticidad del hombre a través de los personajes de su obra posterior a Las armas secretas (1959), que es el libro que incluye el cuento "El perseguidor". Este punto de inflexión lo señala el propio Cortázar en diferentes entrevistas, entre las que se destacan las concedidas a Ernesto González Bermejo (1986).

Sin embargo, aunque lo anterior resuelva parcialmente la pregunta al ofrecer formas de representar la condición humana (muchas de ellas asociadas a los elementos arquetípicos presentes en Poe), no da cuenta de aquello que tiene que ver con la circunstancia del hombre y su relación con el otro. En estos términos, la ambigüedad no termina de resolverse en Cortázar, pues en lugar de sintetizarse, parece crecer, pues no solo se trata de la pregunta por la autenticidad, como lo señala Canclini. En "Del cuento breve y sus alrededores" (1969), Cortázar se pregunta por esa relación entre el hombre y su circunstancia, ya no en lo que se refiere a la construcción del personaje, sino al momento creativo:

¿Cómo describir la atmósfera que antecede y envuelve el acto de escribirlo [el cuento]? Si Poe hubiera tenido ocasión de hablar de eso, estas páginas no serían intentadas, pero él calló ese círculo de su infierno y se limitó a convertirlo en "The Black Cat" o en "Ligeia". No sé de otros testimonios que puedan ayudar a comprender el proceso desencadenante y condicionante de un cuento breve digno de recuerdo; apelo entonces a mi propia situación de cuentista (...) que de pronto, instantáneamente, (...) en la oficina mientras revisa una traducción sospechosa acerca del analfabetismo en Tanzania, deja de ser él-y-sucircunstancia y sin razón alguna, sin preaviso, sin el aura de los epilépticos, sin la crispación que precede a las grandes jaquecas, sin nada que le dé tiempo a apretar los dientes y a respirar hondo, es un cuento, una masa informe sin palabras ni caras ni principio ni fin pero ya un cuento, algo que solamente puede ser un cuento y además en seguida, inmediatamente (...) escribir un cuento así es simultáneamente terrible y maravilloso, hay una desesperación exaltante, una exaltación desesperada; es ahora o nunca, y el temor de que pueda ser nunca exacerba el ahora, lo vuelve máquina de escribir corriendo a todo teclado, olvido de la circunstancia, abolición de lo circundante. Y entonces la masa negra se aclara a medida que se avanza, increíblemente las cosas son de una extrema facilidad como si el 
cuento ya estuviera escrito con una tinta simpática y uno le pasara por encima el pincelito que lo despierta. (...) En esa tregua, la nostalgia introduce una variante en la afirmación de Ortega: hay hombres que en algún momento cesan de ser ellos y su circunstancia, hay una hora en la que se anhela ser uno mismo y lo inesperado, uno mismo y el momento en que la puerta que antes y después da al zaguán se entorna lentamente para dejarnos ver el prado donde relincha el unicornio. ${ }^{5}$ (CORTÁZAR, 1969/2013 p. $40,41,44)$.

Así, pues, la noción del hombre y su circunstancia parece manifestarse claramente en la cotidianidad. Es difícil no apelar a Heidegger como lo hace García Canclini, para hablar del ser-delhombre-en-el-mundo. Y sin embargo, este problema para Cortázar no se resuelve de manera filosófica, sino cuestionando las relaciones que establecemos con el entorno cotidiano, con los otros, pero también con nuestro momento histórico. Este último elemento aparece tardío en la obra de Cortázar, si lo pensamos solo desde una dimensión política, pero siempre estuvo presente si entendemos que cuestionar lo que aceptamos como real significa cuestionar lo que nuestro tiempo rechaza por extraordinario: lo que la ciencia puede explicar hoy, hace doscientos años era fruto de hechicerías o azares inexplicables. El límite entre lo real y lo fantástico nuevamente se emborrona, y con ello modifica las relaciones con nuestro entorno, con otros individuos. De nuevo hay que decir que el problema no es solo temático.

García Canclini señala que la conciencia del ser en el mundo y la complejidad de la relación con el otro, entre otros, son aspectos en los que se observa, en los personajes de Cortázar, una búsqueda de autenticidad. Dicha búsqueda está reforzada por las repercusiones de los acontecimientos del texto literario en su forma; esto se hace evidente en cuentos como "El perseguidor", "Todos los fuegos el fuego", "La flor amarilla", "Axolotl", "El otro cielo", entre otros. Lo que en Poe apunta a la belleza en el poema o la verdad en el cuento, en Cortázar supone la exploración de la forma del cuento como mecanismo para representar esas fracturas, esa puerta que se entorna para mostrarnos que lo que en un momento era el zaguán conocido, es ahora la pradera donde pasta el unicornio. Llama la atención que un problema técnico asociado a la construcción de personajes, que a su vez supone cierta conciencia de nuestra dimensión ética y existencial, pueda resolverse a partir de la pregunta por el momento creativo.

Además, cuando Cortázar habla de aquello que Poe se reserva para sí, se refiere no solo al silencio sobre la dimensión emocional del acto creador, tan importante para el argentino. Se refiere también al problema de la verdad (aunque no lo haga explícitamente). Si el objeto del cuento es la verdad, podemos inferir que ésta tiene que ver con un rasgo ontológico del hombre que solo puede ser expresado por la ficción; Poe alerta: nada de alegorías, el acontecimiento tiene que ser concreto. Y sin embargo, Cortázar añade que ese acontecimiento concreto no importa por su carácter temático, pues los temas en literatura son a la vez múltiples y repetitivos. El tratamiento del acontecimiento es lo que importa, y este tiene que ser intenso. Es en esa intensidad donde radica la posibilidad de abrir la puerta a ese rasgo de nuestro ser en el mundo que solo puede ser observado

\footnotetext{
5 Las cursivas son mías.
} 
de soslayo, como la Gorgona reflejada en el escudo de Perseo.

La intensidad, vale insistir, es algo que tiene que ser construido por el autor. En su conferencia dictada en Casa de Las Américas, y posteriormente publicada bajo el título "Algunos aspectos del cuento" (1971), Cortázar resalta la relación de la intensidad con el acaecimiento, pero también profundiza en su dimensión técnica:

Podemos adelantar ya que las nociones de significación, de intensidad y de tensión han de permitirnos, como se verá, acercarnos mejor a la estructura misma del cuento.

(...) El elemento significativo del cuento parecería residir principalmente en su tema, en el hecho de escoger un acaecimiento real o fingido que posea esa misteriosa propiedad de irradiar algo más allá de sí mismo al punto que un vulgar episodio doméstico (...) se convierta en el resumen implacable de una cierta condición humana, o en el símbolo quemante de un orden social o histórico (CORTÁZAR, 1971, p. 407).

Hasta este punto, Cortázar define el primer elemento en términos temáticos; sin embargo, propone una relación interdependiente, la significación solo funciona cuando se incorporan a su desarrollo la intensidad y a la tensión:

(...) Lo que llamo intensidad en un cuento consiste en la eliminación de todas las ideas o situaciones intermedias, de todos los rellenos o fases de transición que la novela permite e incluso exige. (...) Pero pensemos ahora en los cuentos de Joseph Conrad, de D. H. Lawrence, de Kafka. En ellos, con modalidades típicas de cada uno, la intensidad es de otro orden, y yo prefiero darle el nombre de tensión. Es una intensidad que se ejerce en la manera con que el autor nos va acercando lentamente a lo contado. Todavía estamos muy lejos de saber lo que va a ocurrir en el cuento, y sin embargo no podemos sustraernos a su atmósfera (idem, p. 411-412).

Así, pues, intensidad y tensión confluyen en el desarrollo del acontecimiento del que se ocupa el cuento. Esto es lo que configura la significación del mismo y, en consecuencia, el efecto que puede tener en el lector. En términos técnicos, la función del autor es administrar lo narrado en función de la intensidad de dicho efecto, y para ello se vale de una presentación de los elementos en el relato que sea capaz de generar momentos que aumenten la tensión en el lector, o por el contrario, que le permitan un ritmo de lectura más lento, reflexivo. En todo caso, estos ritmos tienen que ser concebidos en favor del efecto buscado, de la exploración de la significación de la historia, su impresión en el lector.

En el plano de la experiencia estética, encontramos una triple relación que responde a la pregunta por la circunstancia del hombre: en primer lugar, la búsqueda de la autenticidad, es decir, el hombre que, enfrentado a su circunstancia, su manera de ser en el mundo, se pregunta por el sentido de la existencia. De aquí se abre una doble posibilidad: en el plano del autor, dicha indagación le impele a la creación literaria; en el plano del personaje, ésta se expresa a través de lo que le ocurre; el sentido del acontecimiento (cotidiano o extraordinario) del que se ocupa el cuento. Dicho sentido, que se proyecta en términos técnicos como acabé de mencionar, se construye también en relación con una forma de ser en el mundo, que es concebida por el autor. La forma de ser en el mundo del hombre, para Cortázar, supone una condición que puede ser puesta en jaque por la aparición de lo extraordinario, o bien en relación con el 
otro. La irrupción de lo extraordinario en lo real, que para el argentino es la base de su literatura, supone, precisamente, la materialización del enfrentamiento del hombre con su circunstancia (lo que tiene las mismas dimensiones que la idea de verdad en Poe).

Para concluir, más que decir que estas reflexiones no son afirmaciones fijas sobre la creación literaria, es necesario reconocer que están en constante revisión y re-construcción. Las categorías intensidad y tensión, además de ser elementos distintivos de tipos de cuentos, constituyen posibilidades de desarrollo de la forma que no son necesariamente excluyentes (ni se limitan a la literatura fantástica). La obra de cuentistas como Raymond Carver, Flannery O'Connor o Alice Munro dan cuenta de diversas formas de relacionar estos dos elementos. En últimas, cuando hablamos de las lecturas de los maestros, es inevitable pensar en una relación de admiración y deconstrucción de las literaturas heredadas. Un movimiento que se mueve siempre entre la repetición de temas y la apropiación y reinvención de los mecanismos narrativos, en aras de poner en juego una narrativa que se ajuste a las formas de ser en el mundo de las que es contemporánea.

\section{Referencias}

BARTHES, R. S/Z. Buenos Aires: Siglo XXI, 2001. CORTÁZAR, J. El poeta, el narrador y el critico. In: POE, E. A. Obras en prosa. San Juan: Universidad de Puerto Rico, 1956. p. LV-XCVII.

- Del cuento breve y sus alrededores. Último Round. México: R.M., 2013 (1969). Primer piso: p. 35-46.

- Algunos aspectos del cuento. In: Cuadernos Hispanoamericanos, n. 25, p. 403-406, 1971, marzo.

. Cartas. Edición electrónica en formato Epub. Madrid: Alfaguara, 2000.

GARCÍA CANCLINI, N. Cortázar, una antropología poética. Buenos Aires: Nova, 1968.

GONZÁLEZ BERMEJO, E. Revelaciones de un cronopio: conversaciones con Julio Cortázar. Buenos Aires: Contrapunto, 1986.

POE, E. A. Obras en prosa. San Juan: Universidad de Puerto Rico, 1956. p. LV-XCVII.

. Hawthorne. Ensayos y críticas. Madrid: Alianza, 1973. p. 125-141.

WILLIAMS, L. V. La cuentística de Julio Cortázar: Teoría y práctica. Tesis Doctoral - Universidad de Indiana, Indiana, 1974.

Recebido: 9 de maio de 2017. Aceite: 15 de julho de 2017. 